\title{
Traumatic Panniculitis-the Spectrum
}

Jochanan E. Naschitz

Bait Balev Nesher and The Ruth and Bruce Rappaport Faculty of Medicine, Technion, Israel Institute of Technology, Haifa, Israel.

*Corresponding Author: Jochanan E. Naschitz, Bait Balev Nesher and The Ruth and Bruce Rappaport Faculty of Medicine, Technion, Israel Institute of Technology, Haifa, Israel.

Received date: January 11, 2021; Accepted date: March 30, 2021; Published date: April 02, 2021

Citation: Jochanan E. Naschitz. (2021) Traumatic Panniculitis-the Spectrum. International Journal of Clinical Case Reports and Reviews. 6(5); DOI: $10.31579 / 2690-4861 / 106$

Copyright: () 2021 Jochanan E. Naschitz, This is an open-access article distributed under the terms of the Creative Commons Attribution License, which permits unrestricted use, distribution, and reproduction in any medium, provided the original author and source are credited.

\begin{abstract}
Panniculitis, when induced by physical trauma or by chemical agents at injection sites, presents as indurated subcutaneous nodules or plaques. The clinical picture may vary, but the context makes usually the diagnosis easy. Three case histories from our department illustrate the spectrum of traumatic panniculitis: subcutaneous nodules at injection sites, a large subcutaneous mass disproportionate to mild trauma, and, at the other end of the spectrum, severe adipose tissue necrosis with liquefied fat discharging through surface wounds. Traumatic panniculitis is self-limiting and only requires symptomatic treatment.
\end{abstract}

Keywords: panniculitis, physical trauma, injection-site injury, encapsulated fat necrosis, lipoma

\section{Introduction}

Traumatic panniculitis refers to changes in the subcutaneous adipose tissue caused by physical trauma or by chemical agents at injection sites $[1,2]$. The lesions are indurated, warm, subcutaneous plaques or nodules, their occurrence and size not necessary relating with the intensity of injury. There may be areas of fluctuance with liquefied fat discharging through a surface wound. Microscopic examination of a biopsy specimen typically shows microcysts surrounded by histiocytes, foam cells and inflammatory cells. Late lesions may show fibrosis, lipomembranous changes, or dystrophic calcic deposits. The clinical picture is nonspecific, but the context usually makes the diagnosis easy. Traumatic panniculitis is a self-limiting disorder and requires only symptomatic treatment.

The spectrum of mild-to-severe traumatic panniculitis is illustrated in three case histories from our recent experience.

\section{Case histories}

Case 1. Fall, injury, adipose tissue necrosis with liquefied fat discharged through a surface wounds. A 67-year-old woman with renal cell carcinoma metastatic to bones, lungs and brain was referred for hospice care. Six years after nephrectomy and numerous lines of chemotherapy, radiation and immunotherapy, no further oncology treatment was practical. The patient was morbidly obese (BMI 46). She was treated for major depression, hypothyroidism and chronic renal failure. Recently she felt suffering numerous contusions, predominantly of the legs. Subcutaneous nodules and plaques developed at the injured sites as well as skin ulcers. At the time of admission to our ward her body temperature, blood pressure, heart rate, and $\mathrm{SpO}_{2}$ were normal. There were local areas of skin discoloration and fluctuance on the lower legs (Figure 1). Several ulcers had developed on her calves wherefrom liquefied adipose tissue discharged (Figure 2). The haemoglobin was $8.4 \mathrm{~d} / \mathrm{dL}, \mathrm{MCV} 99, \mathrm{WBC}$ $8300 / \mathrm{mm}^{3}$, neutrophiles $87 \%, \mathrm{C}$ reactive protein $8.3 \mathrm{mg} / \mathrm{dL}$, creatinine kinase $32 \mathrm{u} / \mathrm{L}$, eGFR $37 \mathrm{~mL}$, serum creatinine $1.44 \mathrm{mg} / \mathrm{dL}$, albumin 2.9 $\mathrm{g} / \mathrm{dL}$. Her current medications were dexamethasone, venlafaxine, mirtazapine, quetiapine, clonazepam, oxycodone and bisacodyl. The patient's subsequent downhill course was caused by complications of cancer and sepsis.

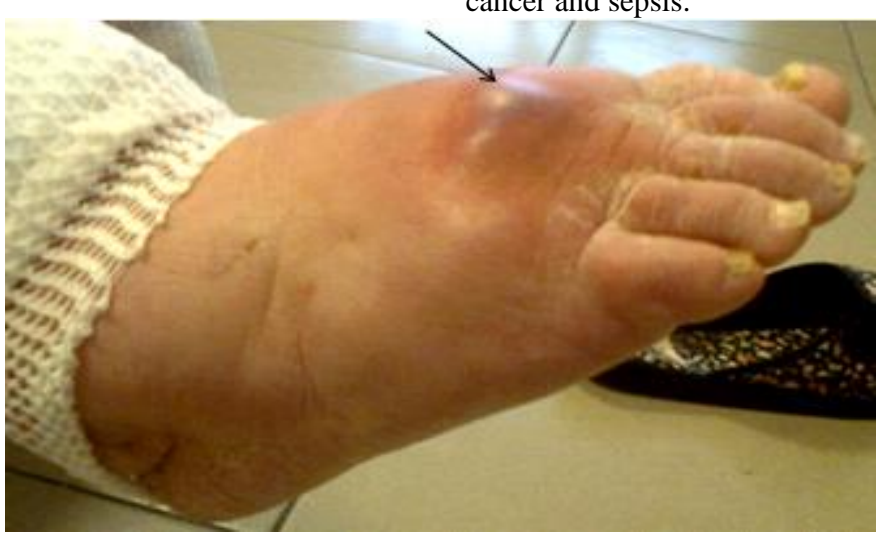




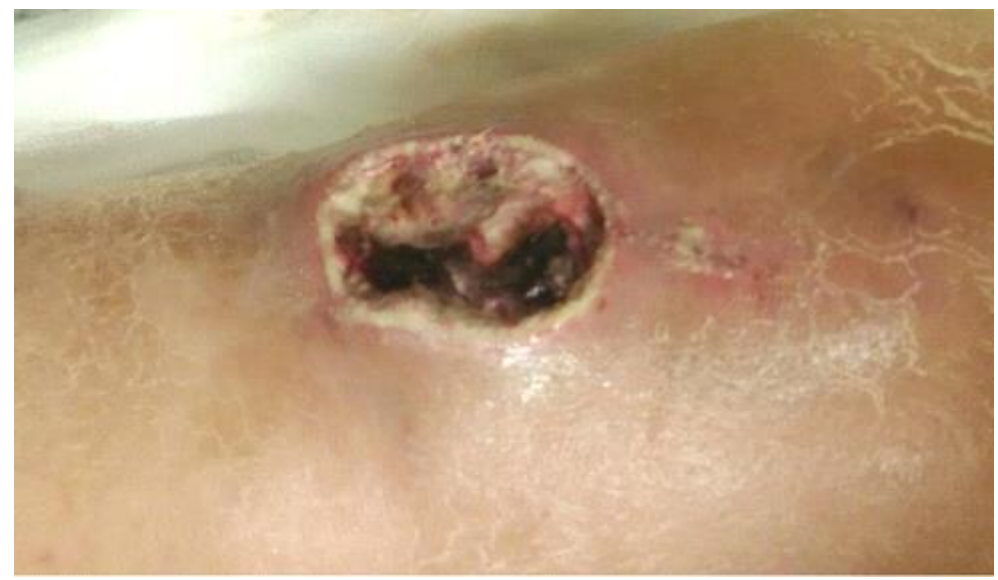

Figure 2: Traumatic panniculitis. The ulcer on the right calf developed after discharge of liquefied adipose tissue through the skin.

Case 2. Immobility, unperceived injury, focal adipose tissue damage. This 90-year-old man in vegetative state was receiving prolonged mechanical ventilation and enteral feeding through gastrostomy. Notable in his medical history were ischemic strokes, right spastic hemiplegia, arterial hypertension and hypothyroidism. He was hemodynamically stable. Except for mild normocytic anemia results of blood cell counts and chemistry laboratory were normal. On physical examination, six years after he was admitted to our ward, a large subcutaneous mass evolved on his paralyzed right forearm (Figure 3). In the given context the diagnosis was traumatic panniculitis, though actual trauma has not been perceived. By protective cushioning of the right forearm the subcutaneous mass diminished (Figure 4) and resolved over a two months period.
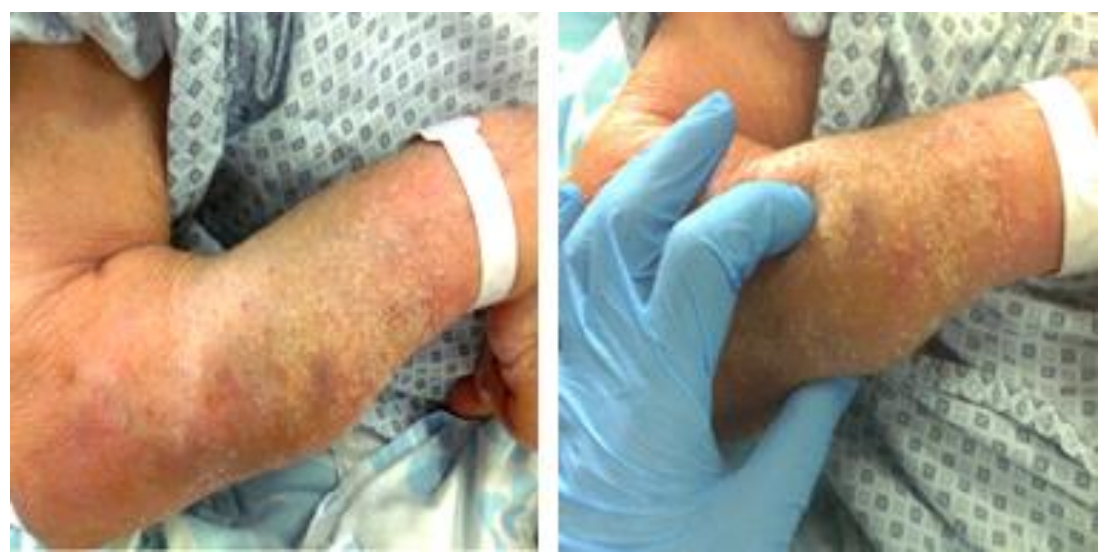

Figure 3: Traumatic panniculitis on the right forearm. Seborrheic dermatitis and mild bruising covered the subcutaneous mass of moderate consistency measuring $8 \times 9 \mathrm{~cm}$ in diameter.

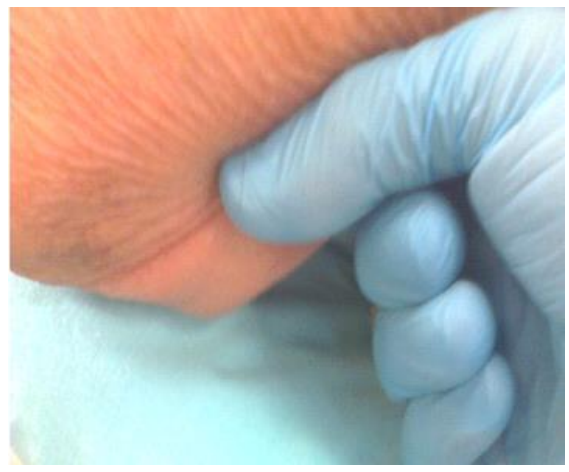

Figure 4: Spontaneous, subtotal resolution of the mass six weeks after diagnosis.

Case 3. Panniculitis at injection sites. This 70-year-old woman was recovering from sepsis, shock, acute renal failure and subsequent massive pulmonary embolism. After thrombectomy enoxaparin treatment was instituted. Her medical history was notable for type 2 diabetes mellitus, morbid obesity and arterial hypertension. At the time of admission to rehabilitation the patient had an oral temperature of $36.8^{\circ} \mathrm{C}$ with a heart rate of 72 beats/min, blood pressure of $107 / 56 \mathrm{mmHg}$ and was breathing comfortably on room air. Abdominal examination showed a nondistended abdomen with normal bowel sounds. Numerous subcutaneous nodules were noticed at injections sites in the abdominal subcutaneous tissue, 1-3 $\mathrm{cm}$ in diameter, some with erythema on the overlying skin. Similar but larger lesions, measuring $10-12 \mathrm{~cm}$ in diameter, were observed on the 
dorsal areas of the upper arms. Pertinent laboratory results included hemoglobin $7.8 \mathrm{~g} / \mathrm{dL}$, WBC 6900/mm3, platelets 355000/mm3, eGFR 54 $\mathrm{ml} / \mathrm{min}$. The patient's medications were enoxaparin $100 \mathrm{mg} /$ day s.c., insulin, bisoprolol fumarat, amiodarone, furosemide, omeprazole, and escitalopram. The subcutaneous nodules had appeared at the injection sites of low-molecular-weight heparin (Figures 5 and 6), while injection of insulin has not induce similar lesions (insulin was well tolerated well over twenty years).
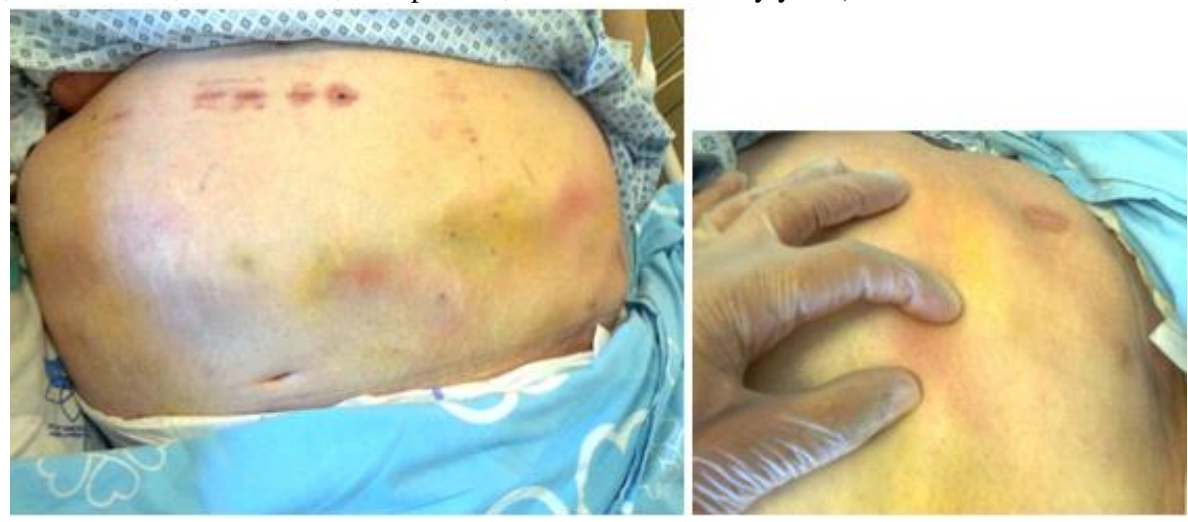

Figure 5: Firm subcutaneous nodules at enoxaparin injection sites in the abdominal wall

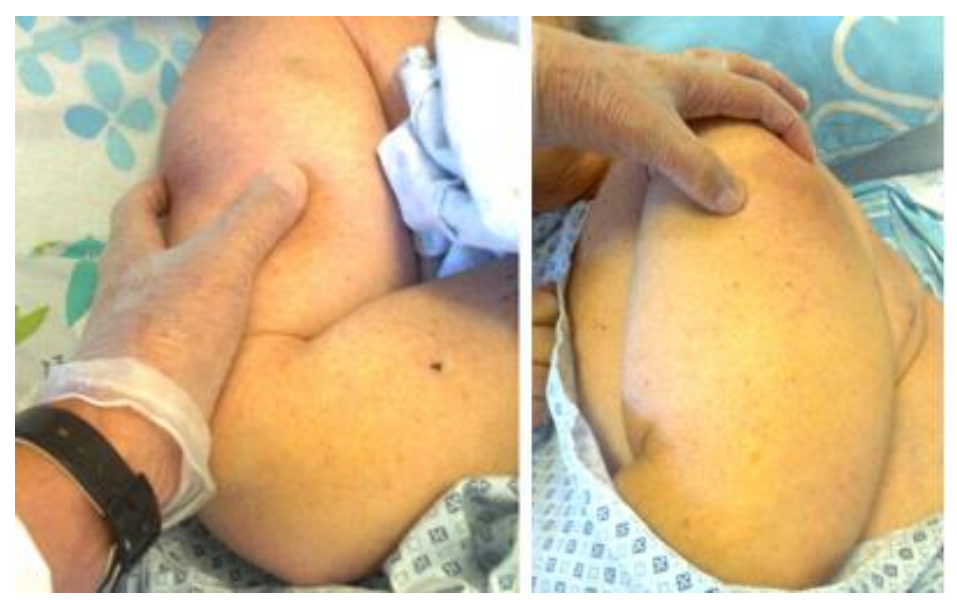

Figure 6: Firm subcutaneous mass at enoxaparin injection site in the upper arms

Enoxaparin was replaced by apixaban whereupon slow resolution of the subcutaneous nodules was observed.

\section{Discussion}

Accidental trauma to the skin may induce necrosis of the subcutaneous adipose tissue. The lesions present as subcutaneous nodules, plaques or masses similar to a lipoma. There may be erythema that develops over top of the area of panniculitis. A prior trauma is often not recalled [1]. The lesions are composed of lobules of necrotic fat surrounded by a thin fibrous capsule. "Encapsulated fat necrosis" is the preferred designation for the small mobile nodules, also called "mobile encapsulated lipoma" and "nodular-cystic fat necrosis". The lesions are whitish-yellow in color and measure 3-20 $\mathrm{mm}$ in diameter but may be as large as $18 \mathrm{~cm}$, reported in an elderly female with morbid obesity [3, 4]. Focal liquefaction of the injured fat often follows, and this is sometimes discharged through a surface wound. Injection-site panniculitis has been described under treatment with insulin, low molecular weight heparin (exemplified in Case 3 of this series), interferon, measles vaccine, exenatide [5-7].

The three cases illustrate a spectrum of mild-to-severe traumatic panniculitis. The diagnosis was simple at the bedside in the appropriate clinical setting. If the diagnosis is uncertain, confirmatory evidence is provided by ultrasonography or CT. The differential diagnosis comprises hematoma and fluid collection. Organizing hematomas with deposition of iron pigments are often found in panniculitis caused by blunt trauma.
There may be similarities with pancreatic panniculitis, $\alpha 1$-antitrypsin deficiency panniculitis, and necrotic soft tissue tumors but the clinical scenario differs. Post-surgical panniculitis is a lobular foam cell panniculitis characterized by simultaneous dermal and hypodermal changes, expression of the tissue response to a surgical trauma. It represents a variant of lobular panniculitis and is characterized histologically by pseudocystic degeneration and necrosis of adipocytes, the presence of foam cells (lipophages) and extravasation of erythrocytes [4].

Factitial panniculitis may follow injections of all manner of substances into the subcutaneous adipose tissue: milk, urine, feces, oils (oleoma), and drugs. It may follow a secondary infection of tattoo. The clinical manifestations will depend on the nature and site of the insult. Cupping and other trauma may also induce a panniculitis [8]. A paraffinoma is a special form of factitial panniculitis resulting from the injection of paraffin, into the subcutaneous tissue, particularly in an attempt to produce enlargement of the penis or the breasts. Other oils and silicones have been involved. Grease gun granuloma with panniculitis results from the accidental firing of this device, which is used especially by auto mechanics. A case masquerading as pyoderma gangrenosum has been reported.

Three case histories from our department illustrate the variety of traumatic panniculitis: subcutaneous nodules at injection sites, a large subcutaneous mass after a mild unperceived trauma, and, at the other end 
of the spectrum, severe adipose tissue necrosis with liquefied fat discharging through surface wounds. In all cases, traumatic panniculitis was self-limiting.

\section{References}

1. James WD, Elston DK, Treat JR, Rosenbach MA, Neuhaus IM. (2020) Diseases of Subcutaneous Fat. In: Andrews' Diseases of the Skin. 23; 485-495.

2. Moreno A, Marcoval J, Peyri J. (2008) Traumatic panniculitis. Dermatol Clin. 26:481-483.

3. Hurt MA, Santa Cruz DJ. (1989) Nodular-cystic fat necrosis. A reevaluation of the so-called mobile encapsulated lipoma. J Am Acad Dermatol. 21(3 Pt 1):493-498.
4. Patterson JW. (2021) Panniculitis .Weedon's Skin Pathology. 18:565-589.

5. Koch P, Munssinger T, Rupp-John C, Uhl K. (2000) Delayed-type hyper sensitivity skin reactions caused by subcutaneous unfractionated and low-molecular-weight heparins: Tolerance of a new recombinant hirudin. J Am Acad Dermatol. 42:612-619.

6. Funt SA, Hidalgo A, Panicek DM. (2002) Subcutaneous nodules at the injection site of low-molecular-weight heparin: a mimic of metastatic disease at CT. J Comput Assist Tomogr. 26:520-523.

7. Ozuguz P, Kacar SD, Karaca S, Tokuol C. (2014) A case of septal panniculitis secondary to interferon treatment. Cutan Ocul Toxicol. 33:351-352.

8. Boyd AS. (2014) Revision: cutaneous Munchausen syndrome. J Cutan Pathol. 41:333-336. 